\title{
Line formation regions of the UV spectrum of Cl Cygni
}

\author{
J. Mikołajewska ${ }^{1}$, M. Friedjung ${ }^{2}$, and C. Quiroga ${ }^{3,4}$ \\ 1 Nicolaus Copernicus Astronomical Center, Bartycka 18, 00-716 Warsaw, Poland \\ e-mail: mikolaj@camk.edu.pl \\ 2 Institut d'Astrophysique de Paris - UMR 7095, CNRS/Université Pierre et Marie Curie, 98 bis Boulevard Arago, 75014 Paris, \\ France \\ 3 Consejo Nacional de Investigaciones Científicas y Técnicas de la República Argentina (CONICET) \\ ${ }^{4}$ Facultad de Ciencias Astronómicas y Geofísicas, Universidad Nacional de La Plata, La Plata, Argentina
}

Received 7 January 2006 / Accepted 22 August 2006

\section{ABSTRACT}

\begin{abstract}
Aims. They are the interpretation of the emission line formation regions in CI Cygni Methods. They involve the examination of radial velocities and fluxes of ultraviolet emission lines at different epochs, deduced from archival IUE and GRHS/HST spectra.

Results. The line fluxes give electron densities and were in addition used to calculate emission measures, suggesting line formation in regions rather smaller than the binary separation. Examination of the radial velocities led to us to find a systematic redshift of the high ionization resonance lines with respect to the intercombination, and He II lines. Possible explanations of the redshift and the high resolution GHRS C IV profile are discussed. We favour that involving resonance line absorption by a circum-binary region most probably in an asymmetric wind interaction shell or in a wind from the accretion disk.
\end{abstract}

Key words. stars: binaries: symbiotic - stars: fundamental parameters - stars: mass-loss - stars: individual: CI Cyg - line: profiles

\section{Introduction}

CI Cyg is a symbiotic binary, containing a cool giant, of type M5.5 (Mürset \& Schmid 1999), and a much more compact companion. The infrared spectrum shows no sign of circumstellar dust. It is moreover particularly fruitful to study CI Cyg, because it undergoes eclipses. A detailed investigation of this object was performed by Kenyon et al. (1991). They concluded that the compact object was a main sequence star of $0.5 M_{\odot}$, surrounded by a large accretion disk. Recent work shows however that the compact component of this binary may not be a main sequence star (e.g. Mikołajewska 2003).

CI Cyg is one of the very few symbiotic binaries in which the cool giant fills or nearly fills its Roche lobe (e.g. Mikołajewska 2001, and references therein). CI Cyg is also one of the very few symbiotic systems for which a high quality radio spectrum, covering the range between $6 \mathrm{~cm}$ and $850 \mu \mathrm{m}$, has been obtained (Mikołajewska \& Ivison 2001). These radio data enabled them to determine the free-free turnover frequency of the ionized component, and to critically test the known models for radio emission from symbiotic stars. Unfortunately, they ruled out the two most popular models: ionization of the red giant wind by Lyman continuum photons from its hot companion, and emission resulting from the interaction of winds from the two binary components.

A systematic shift in radial velocity between ultraviolet intercombination lines and ultraviolet high ionization permitted resonance emission lines in symbiotic binary spectra, was found by Friedjung et al. (1983). The latter are generally redshifted with respect to the former, and Mikołajewska \& Friedjung (2005) have recently reported an orbital variation of this redshift in CI Cyg. Unlike the intercombination lines, the resonance lines are expected to be optically very thick. Their redshift might be due to the absorption of both the continuum and part of the emission line by P Cygni profile absorpion components, or only to a radiative transfer effect produced by photon scattering in an expanding medium.

In the present work, we have examined in detail the effect for the symbiotic binary, CI Cyg, and we find some support for the P Cygni profile interpretation. Our data base is presented in Sect. 2, analyzed in Sect. 3, and the results discussed in Sect. 4. We conclude with a brief summary of these results in Sect. 5 .

\section{Observations}

The log of observations used is shown in Table 1. They include high spectral resolution observations made with the IUE satellite, archived in the INES data base and higher resolution GHRS/HST observations made on 1993 October, 11 in the regions of different lines. Though the latter were made before the COSTAR correction for the spherical aberration of the mirror, the effect should not be large, as the Small Science Aperture was used $\left(0.25^{\prime \prime}\right)$. Profiles are shown in Fig. 1. Radial velocities and fluxes of different lines were obtained by a Gaussian fit of the line profile (Tables 2, 3, and 4). Radial velocity curves are shown in Fig. 2.

\section{Results and interpretation}

\subsection{Radial velocity curves and emission line flux changes}

He II traces the motion of the hot component but the systemic velocity, $\gamma=0.6 \pm 1.7 \mathrm{~km} \mathrm{~s}^{-1}$, seems to be blueshifted by about $18 \mathrm{~km} \mathrm{~s}^{-1}$ with respect to the $\mathrm{M}$ giant orbital solution of Kenyon et al. (1991). A similar blueshift in the systemic velocity was found for the radial velocity curve for the optical He II $4686 \AA$ 
Table 1. Log of the UV observations of CI Cyg.

\begin{tabular}{lcccc}
\hline \hline Image No. & Date & MJD & Phase $^{1}$ & $T_{\exp }[\mathrm{s}]$ \\
\hline SWP 06818 & Dec. 11, 1979 & 44157 & 0.718 & 5400 \\
SWP 09256 & Jun. 6, 1980 & 44401 & 0.003 & 10800 \\
SWP 13795 & Apr. 24, 1981 & 44719 & 0.375 & 4800 \\
SWP 14754 & Aug. 14, 1981 & 44831 & 0.506 & 5400 \\
SWP 18602 & Nov. 20, 1982 & 45294 & 0.046 & 40800 \\
SWP 37047 & Sep. 17, 1989 & 47786 & 0.959 & 6480 \\
SWP 45102 & Jul. 9, 1992 & 48812 & 0.159 & 5400 \\
SWP 50760 & May 14, 1994 & 49486 & 0.946 & 6300 \\
SWP 51109 & Jun. 16, 1994 & 49520 & 0.986 & 23640 \\
SWP 52838 & Nov. 20, 1994 & 49676 & 0.168 & 16200 \\
SWP 54471 & Apr. 20, 1995 & 49827 & 0.345 & 21600 \\
SWP 55894 & Sep. 10, 1995 & 49970 & 0.513 & 18000 \\
\hline Z1705107M & Oct. 11, 1993 & 49272 & 0.696 & 1795 \\
Z1705109T & Oct. 11, 1993 & 49272 & 0.696 & 707 \\
Z170510BT & Oct. 11, 1993 & 49272 & 0.696 & 816 \\
Z170510EM & Oct. 11, 1993 & 49272 & 0.696 & 816 \\
Phase calculated from Min $\left(m_{\mathrm{pg}} / B\right)$ & $=$ JD 2442687.1 & $+855.6 \times E$ \\
Belczyński et al. 2000). & \multicolumn{4}{l}{}
\end{tabular}

Table 2. Emission line fluxes and radial velocities from GHRS spectra $(\mathrm{MJD}=49271 / \phi=0.696)$.

\begin{tabular}{ccc}
\hline \hline Line ID & Flux $^{1}$ & $v_{\text {rad }}\left[\mathrm{km} \mathrm{s}^{-1}\right]$ \\
\cline { 2 - 3 } Si IV 1393.755 & 28 & 34 \\
Si IV 1402.769 & 19 & 29 \\
O IV] 1397.166 & 3 & 35 \\
O IV] 1399.731 & 7 & 25 \\
O IV] 1401.115 & 36 & 26 \\
O IV] 1404.740 & 15 & 33 \\
O IV] 1407.333 & 9 & 26 \\
S IV] 1406.004 & 5 & 23 \\
S IV] 1416.872 & 4 & 20 \\
C IV 1548.202 & 350 & 32 \\
C IV 1550.774 & 234 & 30 \\
He II 1640.470 & 205 & 12 \\
O I] 1641.310 & 6 & 14 \\
O III] 1660.800 & 25 & 20 \\
O III] 1666.150 & 68 & 18 \\
Mg V 2783.03 & 21 & 89 \\
Mg II 2796.352 & 30 & 39 \\
Mg II 2803.531 & 22 & 35 \\
\hline $\mathrm{cm}^{-2}$ &
\end{tabular}

${ }^{1} 10^{-14} \mathrm{erg} \mathrm{s}^{-1} \mathrm{~cm}^{-2}$.

line (Kenyon et al. 1991). The amplitude of the circular orbit, $K_{\mathrm{h}}=17.3 \pm 2.8 \mathrm{~km} \mathrm{~s}^{-1}$, combined with that of the $\mathrm{M}$ giant, $K_{\mathrm{g}}=6.7 \pm 0.8 \mathrm{~km} \mathrm{~s}^{-1}$ implies that $q=M_{\mathrm{g}} / M_{\mathrm{h}}=2.58_{0.65}^{0.82}$, and is consistent with other estimates (see Kenyon et al. 1991 for details). We should note in addition that He II shows no systematic shift in radial velocity between decline and quiescence.

The intercombination lines are roughly in phase with the hot component. However the amplitude, $K \sim 10 \mathrm{~km} \mathrm{~s}^{-1}$ is much lower, the systemic velocity, $\sim 10 \mathrm{~km} \mathrm{~s}^{-1}$, is blueshifted with respect to the systemic velocity of the red giant, $\gamma_{\mathrm{g}}=$ $18.4 \pm 0.4 \mathrm{~km} \mathrm{~s}^{-1}$ (Kenyon et al. 1991), and the orbit seems to be eccentric with $e \sim 0.4$, the longitude of periastron, $\omega \sim 0^{\circ}$, and the spectroscopic conjunction occurs at $\phi \sim 0.8$. In fact, they follow with very good precision the orbit found for the optical Fe II emission lines by Kenyon et al. (1991). The solid line in the middle panel of Fig. 2 corresponds to an orbital solution with the same parameters as those for Fe II in Table 8 of Kenyon et al. (1991) except for the amplitude and the systemic velocity which

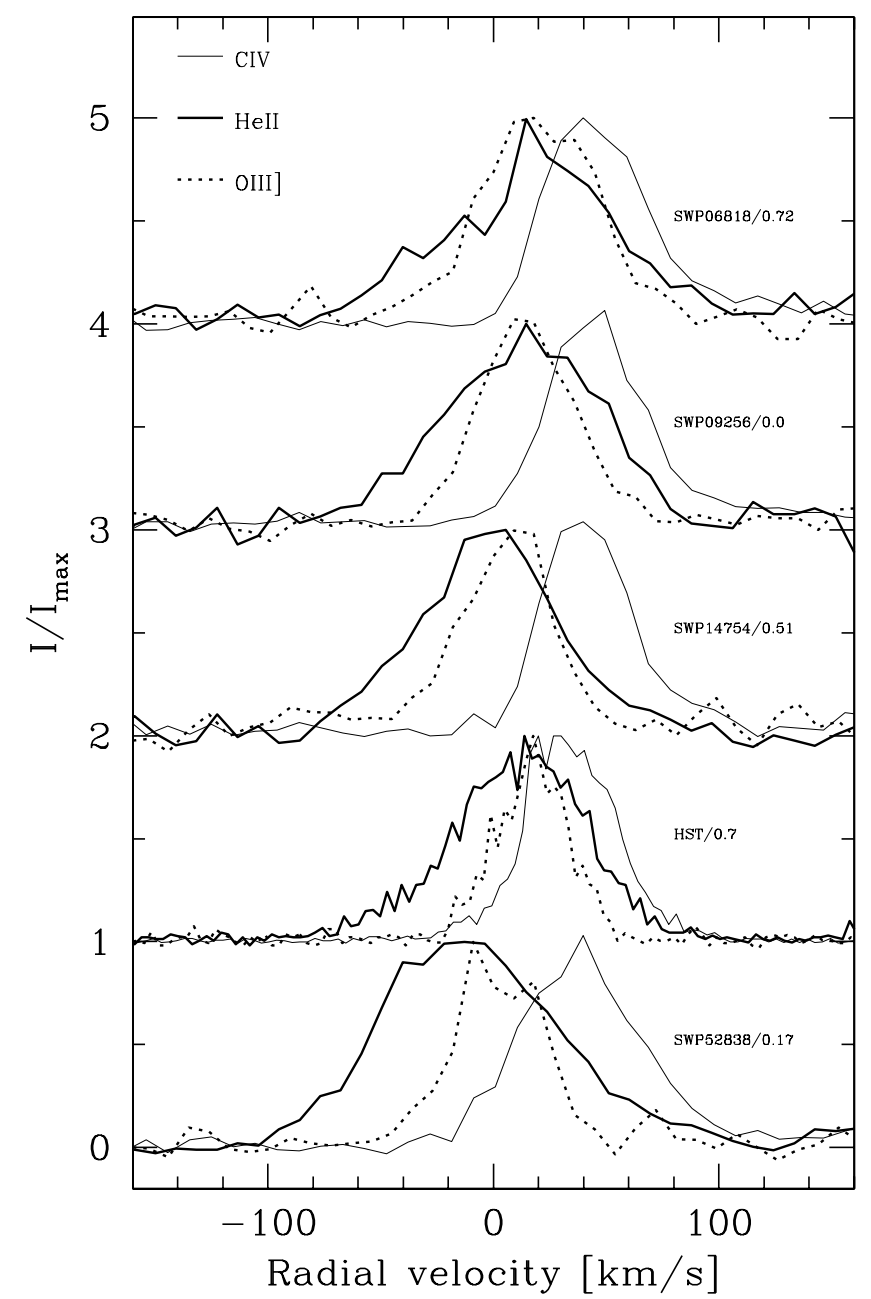

Fig. 1. Examples of emission line profiles of He II $1640 \AA$ A C IV $1548 \AA$ and O III] $1666 \AA$ in CI Cyg. The numbers on the right side refer to the number of the spectrum and orbital phase, respectively.

were fitted with our quiescent data. There is also an interesting systematic difference between the radial velocity curve formed by the quiescent (after 1983), and late decline (1979-82) data, respectively (Fig. 2): although both data sets seem to follow the same orbit the data from the decline are slightly blueshifted (by $\sim 7 \pm 2 \mathrm{~km} \mathrm{~s}^{-1}$ ) with respect to the quiescent data. The only point above the solid curve in Fig. 2 is the measurement from the spectrum obtained in the midle of the 1980 eclipse. We note that the radial velocities for the other emission lines derived from this spectrum are redshifted by $\sim 20 \mathrm{~km} \mathrm{~s}^{-1}$ with respect to the data obtained near the other eclipses, and it may be due to uncertainty in the wavelength calibration.

The blueshift of the systemic velocity of both the optically thin intercombination lines and the usually not optically very thick He II $1640 \AA$ A line suggests line formation regions approaching the observer whereas the systemic velocity difference between the intercombination lines and He II $1640 \AA$, appears to indicate a velocity stratification and/or acceleration effects in the ionized nebula, with electron temperature variations perhaps affecting collisionally excited lines. Probably for the same reason, the He II line shows a systematically broader profile than the intercombination O III line (Fig. 1).

The radial velocities of the optically thick resonance lines are almost stationary, and redshifted by $\sim 10-20 \mathrm{~km} \mathrm{~s}^{-1}$ with 
Table 3. Emission line fluxes from IUE high resolution spectra (in units of $10^{-14} \mathrm{erg} \mathrm{s}^{-1} \mathrm{~cm}^{-2}$ ).

\begin{tabular}{|c|c|c|c|c|c|c|c|c|c|c|c|}
\hline MJD & $\phi$ & $\begin{array}{c}\mathrm{NV} \\
1238 / 42\end{array}$ & $\begin{array}{c}\text { Si IV } \\
1394 / 1403\end{array}$ & $\begin{array}{c}\text { O IV] } \\
1400 / 01 / 05\end{array}$ & $\begin{array}{l}\text { NIV] } \\
1486\end{array}$ & $\begin{array}{c}\text { C IV } \\
1548 / 50\end{array}$ & $\begin{array}{l}\mathrm{He} \text { II } \\
1640\end{array}$ & $\begin{array}{c}\mathrm{O} \mathrm{III]} \\
1660 / 66\end{array}$ & $\begin{array}{c}\text { N III] } \\
1749 / 50 / 52 / 54\end{array}$ & $\begin{array}{l}\mathrm{Si} \text { III] } \\
1892\end{array}$ & $\begin{array}{l}\text { C III] } \\
1909\end{array}$ \\
\hline 44157 & 0.718 & 100/37: & $63 / 52$ & $40 / \ldots / 21$ & 180 & $800 / 588$ & 336 & $35 / 123$ & $40 / 133 / 41 / \ldots$ & 248 & $577 \mathrm{~s}$ \\
\hline 44401 & 0.003 & $25 / \ldots$ & $48 / 44$ & .../.../... & 82 & $489 / 345$ & 137 & $23 / 156$ & $18 / 76 / 41 / \ldots$ & 90 & $\mathrm{~S}$ \\
\hline 44719 & 0.375 & $68: / \ldots$ & $37: / 42:$ & $\ldots / \ldots / \ldots$ & 180 & $410 / 271$ & 336 & $45 / 197$ & $30 / 65 / \ldots / \ldots$ & 99 & 331 \\
\hline 44831 & 0.506 & $64 / 41$ & $41 / \ldots$ & .../.../... & 166 & $350 / 249$ & 360 & $54 / 185$ & $\ldots / 64 / \ldots / \ldots$ & 127 & 443 \\
\hline 45294 & 0.046 & $39 / 21$ & $17 / 12$ & $\ldots / \ldots / \ldots$ & 50 & $\mathrm{~S} / \mathrm{S}$ & $\mathrm{S}$ & $16 / 71 \mathrm{~s}$ & $\ldots / 32 / \ldots / \ldots$ & $\mathrm{S}$ & $\mathrm{S}$ \\
\hline 47786 & 0.959 & $\ldots / \ldots$ & $\ldots / \ldots$ & $\ldots / \ldots / \ldots$ & 39 & $236 / 212$ & 289 & $6 / 50$ & $\ldots / 18 / \ldots / 22$ & 37 & 114 \\
\hline 48812 & 0.159 & 50:/30: & .../... & $\ldots / \ldots / \ldots$ & 135 & $266 / 189$ & 557 & $54 / 123$ & $\ldots / 65 / \ldots / \ldots$ & 62 & 209 \\
\hline 49486 & 0.946 & $36: / 31:$ & 38:/21: & $\ldots / \ldots / \ldots$ & 65 & $401 / 350$ & 419 & .../60 & $\ldots / 38 / \ldots / \ldots$ & 33 & 155 \\
\hline 49520 & 0.986 & $59 / \ldots$ & $28 / \ldots$ & .../.../16 & 52 & $\mathrm{~S} / 268$ & $300 \mathrm{~s}$ & $5: / 67$ & $\ldots / 16 / \ldots / \ldots$ & 26 & $110 \mathrm{~s}$ \\
\hline 49676 & 0.168 & $100 / 64$ & 26:/11: & $17 / \ldots / 23$ & 175 & $327 / 249$ & 583 & $40 / 142$ & $12 / 47 / 12 / 20$ & 58 & $200 \mathrm{~s}$ \\
\hline 49827 & 0.345 & $332 / 178$ & $99 / 61$ & $11 / 95 / 35$ & $221 \mathrm{~s}$ & $\mathrm{~S} / \mathrm{S}$ & $480 \mathrm{~s}$ & $82 / 220 \mathrm{~s}$ & $28 / 67 / 13 / 12$ & $\mathrm{~S}$ & $\mathrm{~S}$ \\
\hline 49970 & 0.513 & $389 / 205$ & $120 / 69$ & $22 / \ldots / 55$ & 279 & $\mathrm{~S} / \mathrm{S}$ & $620 \mathrm{~s}$ & $115 / 304$ & $28 / 82 / 22 / 22$ & $110 \mathrm{~s}$ & $\mathrm{~S}$ \\
\hline
\end{tabular}

s - a few pixels saturated; S - saturated; “..." - too noisy; “.” - uncertain.

Table 4. Radial velocities of the IUE emission lines (in units of $\mathrm{km} \mathrm{s}^{-1}$ ).

\begin{tabular}{|c|c|c|c|c|c|c|c|c|c|c|c|}
\hline MJD & $\phi$ & $\begin{array}{c}\mathrm{NV} \\
1238 / 42\end{array}$ & $\begin{array}{c}\text { Si IV } \\
1394 / 1403\end{array}$ & $\begin{array}{c}\text { O IV] } \\
1400 / 01 / 05\end{array}$ & $\begin{array}{l}\text { N IV] } \\
1486\end{array}$ & $\begin{array}{c}\text { C IV } \\
1548 / 50\end{array}$ & $\begin{array}{l}\mathrm{He} \text { II } \\
1640\end{array}$ & $\begin{array}{c}\text { O III] } \\
1660 / 66\end{array}$ & $\begin{array}{c}\text { N III] } \\
1749 / 50 / 52 / 54\end{array}$ & $\begin{array}{l}\mathrm{Si} \text { III] } \\
1892\end{array}$ & $\begin{array}{l}\text { C III] } \\
1909\end{array}$ \\
\hline 44157 & 0.718 & $37 / 35:$ & $36 / 42$ & $39 / \ldots / 44$ & 26 & $43 / 42$ & 19 & $20 / 20$ & $19 / 14 / 8 / \ldots$ & 21 & 15 \\
\hline 44401 & 0.003 & $47 / \ldots$ & $38 / 41$ & .../.../.. & 19 & $44 / 40$ & 14 & $26 / 14$ & $9 / 10 / 13 / \ldots$ & 12 & 10 \\
\hline 44719 & 0.375 & $27: / \ldots$ & $36: / 32:$ & .../.../... & 8 & $39 / 35$ & -6 & $6 / 7$ & $-9: / 10 / \ldots / \ldots$ & 11 & 2 \\
\hline 44831 & 0.506 & $19 / 22$ & $26 / \ldots$ & $\ldots / \ldots / \ldots$ & 4 & $40 / 39$ & -2 & $11 / 7$ & $\ldots / 4 / \ldots / \ldots$ & 10 & 4 \\
\hline 45294 & 0.046 & $27 / 20$ & $23 / 27$ & $\ldots / \ldots / \ldots$ & 2 & $38 / 29$ & 2 & $-4 / 0$ & $\ldots /-8 / \ldots / \ldots$ & -5 & -1 \\
\hline 47786 & 0.959 & .../... & $\ldots / \ldots$ & .../.../... & 1 & $27 / 25$ & 3 & $3 / 3$ & $\ldots / 3 / \ldots / 5$ & 6 & 4 \\
\hline 48812 & 0.159 & 21:/21: & $\ldots / \ldots$ & .../../... & -3 & $33 / 34$ & -17 & $3 / 3$ & $\ldots / 1 / \ldots / \ldots$ & 8 & 0 \\
\hline 49486 & 0.946 & 15:/24: & 9:/27: & .../.../.. & 7 & $32 / 29$ & 10 & $\ldots / 2$ & $\ldots / 4 / \ldots / \ldots$ & 1 & 9 \\
\hline 49520 & 0.986 & $21 / \ldots$ & $22 / \ldots$ & .../.../12 & -3 & $29 / 22$ & 2 & $\ldots /-8$ & $\ldots /-3 / \ldots / \ldots$ & -4 & -2 \\
\hline 49676 & 0.168 & $33 / 31$ & $42 / 35$ & $-1 / \ldots / 4$ & 2 & $35 / 35$ & -12 & $1 / 2$ & $4 / 3 /-1 / 7$ & 7 & 3 \\
\hline 49827 & 0.345 & $16 / 17$ & $25 / 19$ & $2 / 7 / 13$ & 2 & $31 / 27$ & -23 & $0 / 6$ & $6 / 4 / 0 / 1$ & 8 & 3 \\
\hline 49970 & 0.513 & $33 / 29$ & $35 / 31$ & $14 / \ldots / 31$ & 17 & $39 / 34$ & -4 & $18 / 16$ & $17 / 21 / 11 / 19$ & 25 & 22 \\
\hline
\end{tabular}

“...” - too noisy; “:” - uncertain; for saturated lines the radial velocities (itallic) were obtained by fitting their wings.

respect to the systemic velocity of the red giant. Mikołajewska $\&$ Friedjung (2005) showed that the velocity difference between the resonance and intercombination emission lines seems to follow the radial velocity of the cool giant, with a comparable amplitude, $\sim 6 \mathrm{~km} \mathrm{~s}^{-1}$, and average velocity, $\sim 21 \mathrm{~km} \mathrm{~s}^{-1}$, almost the same as the systemic velocity. We note however that this effect may not be physical because of the apparent constancy of the resonance line radial velocity; which means that the radial velocity difference curve is a mirror image of the radial velocity curve for the intercombination lines.

A comparison of the C IV emission line profiles with those of He II (Fig. 1), and particularly the best resolution profiles taken with the HST (Fig. 3) shows that the red wings of both lines overlap whereas the blue wing of the C IV line seems to be missing, possibly due to the presence of a blue shifted absorption component in a $\mathrm{P}$ Cygni profile. The corrected emission component might in that case trace the motion of the hot component. A P Cygni profile of this sort seems to be mostly related to the hot component's activity: it was certainly more pronounced in the early 1980's (decline from the 1975 outburst) than in the 1990's when the system had reached the quiescent state. A weak absorption component seems to be also present in the blue wing of the He II profile taken in $1979(\phi \sim 0.7)$ whereas it may be marginally visible in the HST/GHRS profile taken in 1993.

It seems that some radial velocities are related to the level of ionization: the HST data give radial velocities of $29 \mathrm{~km} \mathrm{~s}^{-1}$ for the intercombination O IV], $13 \mathrm{~km} \mathrm{~s}^{-1}$ for the intercombination $\mathrm{O} \mathrm{III}$ ] and $11 \mathrm{~km} \mathrm{~s}^{-1}$ for the $\mathrm{OI}$ ] lines. However, there is no systematic difference in the velocities of C IV and Si IV.
The resonance line ratios indicate moderate optical depth effects. In particular, the average ratios are: $F(\mathrm{~N}$ V 1238/1240) $=$ $1.9 \pm 0.3, F(\operatorname{Si}$ IV $1394 / 1403)=1.7 \pm 0.2(1.5$ for HST spectra), and $F(\mathrm{C}$ IV $1548 / 1550)=1.33 \pm 0.05$ (1.5 for HST spectra), respectively. These ratios do not seem to vary with the orbital phase nor the activity, although the line fluxes show both orbitally related and secular changes (Fig. 4). In particular, the permitted resonance and He II 1640 line fluxes were increasing as the system declined from the last outburst, and then stabilized at some high level whereas the intercombination line fluxes tended to decrease. Both the permitted and intercombination line fluxes change with the orbital phase, and the minima in all lines but He II are much deeper and broader in quiescence than during the decline in activity. The same effect is present for the optical Balmer lines.

The observed orbitally related changes of the emission line fluxes require the main line formation regions to be located inside the binary system, with possible stratification. The fact that the resonance lines show practically the same secular trend and eclipse behaviour as the He II line suggests that they are formed in the same region, and that their profiles should be intrinsically the same. Thus the lack of orbitally related radial velocity changes together with their redshift with respect to the He II and intercombination lines indicates that the resonance line profile must be seriously affected by an absorption component. Moreover, the emission must be absorbed outside the binary system in very extended region. The He II $1640 \AA$ line would be not affected by this circumstellar absorption because it is the recombination line from a high excitation level $(41 \mathrm{eV})$. 


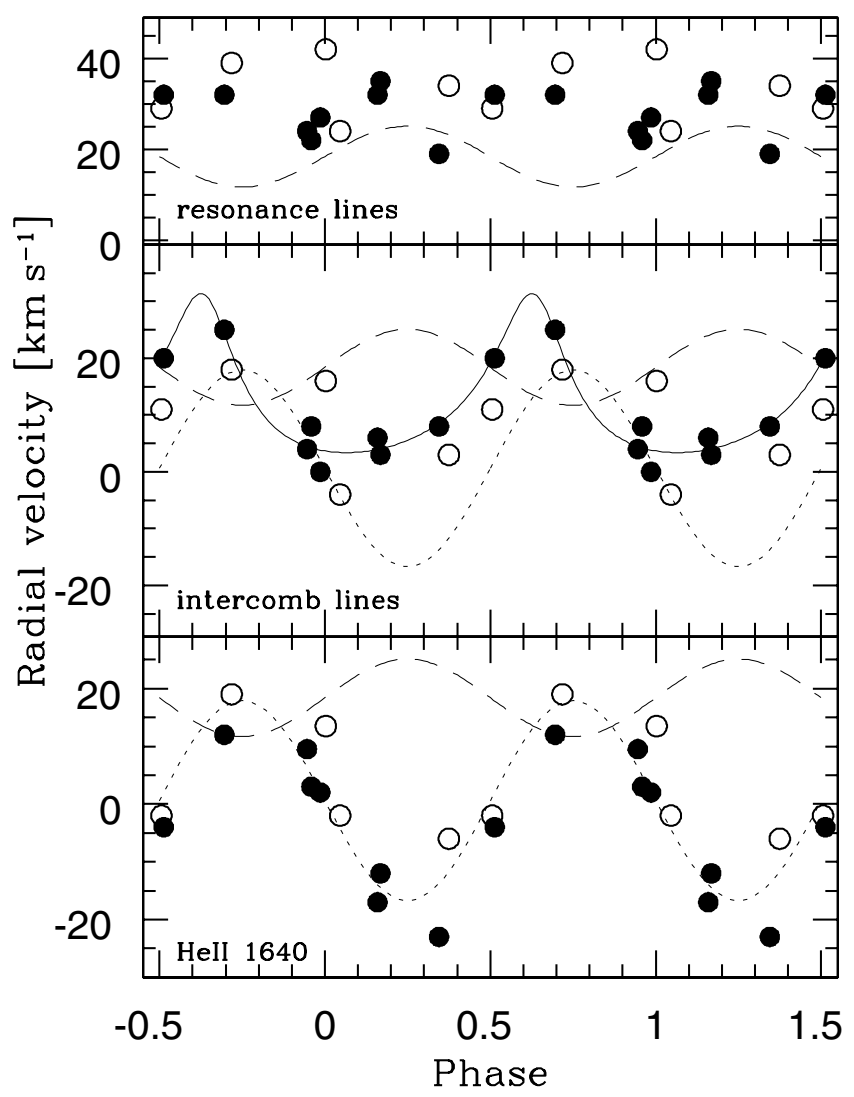

Fig. 2. Radial velocity curves for CI Cyg. The dashed line repeats the orbit of the $\mathrm{M}$ giant and the dotted line - the circular orbit solution for the He II $1640 \AA$ A emission line, respectively. The solid line corresponds to the elliptical orbit solution for the intercombination line (see text for details). Open and closed symbols correspond to the late decline (1979-82) and quiescent data (after 1983), respectively.

The O III] line ratio, the average $F(1666 / 1660)=3.85 \pm 0.45$, is always bigger than the optically thin value, and the effect is much stronger during the eclipse of the hot component. The effect was also stronger before 1983 when CI Cyg was still declining from the large 1975 outburst. If this anomalous line ratio is due to Bowen pumping of optically thick lines as proposed by Kastner et al. (1989), the pumping must be more effective during the decline as well as in the region(s) visible during the eclipse. The fact that the out-of-the-eclipse intensity of the Bowen lines $3133 \AA$ and $2837 \AA$ decreased after 1983 whereas the intercombination doublet itself remained practically constant, suggests that the Bowen excitation rate was indeed higher before 1983 (Kenyon et al. 1991) than in the 1990's. On the other hand, both the Bowen lines and the intercombination doublet show an eclipse effect, although the effect is less pronounced for the Bowen lines.

\subsection{Emission measures}

The density sensitive intercombination line ratios measured on IUE spectra indicate $n_{\mathrm{e}} \sim 4 \times 10^{9}$ and $\sim 3 \times 10^{9} \mathrm{~cm}^{-3}$ for N III] and O IV] (1404.8:1401.2), respectively (Nussbaumer \& Storey 1979; Nussbaumer \& Storey 1982). We note, however, that the O IV] 1404.8 line is blended with S IV] line, which contributes $\sim 10 \%$ of the total line flux. The O IV] line ratios measured on the HST spectrum indicate $n_{\mathrm{e}} \sim 2 \times 10^{9}(1399.8: 1401.2: 1404.8)$ and $\$ 10^{10} \mathrm{~cm}^{-3}$ (1401.2:1407.4), respectively (Harper et al. 1999;

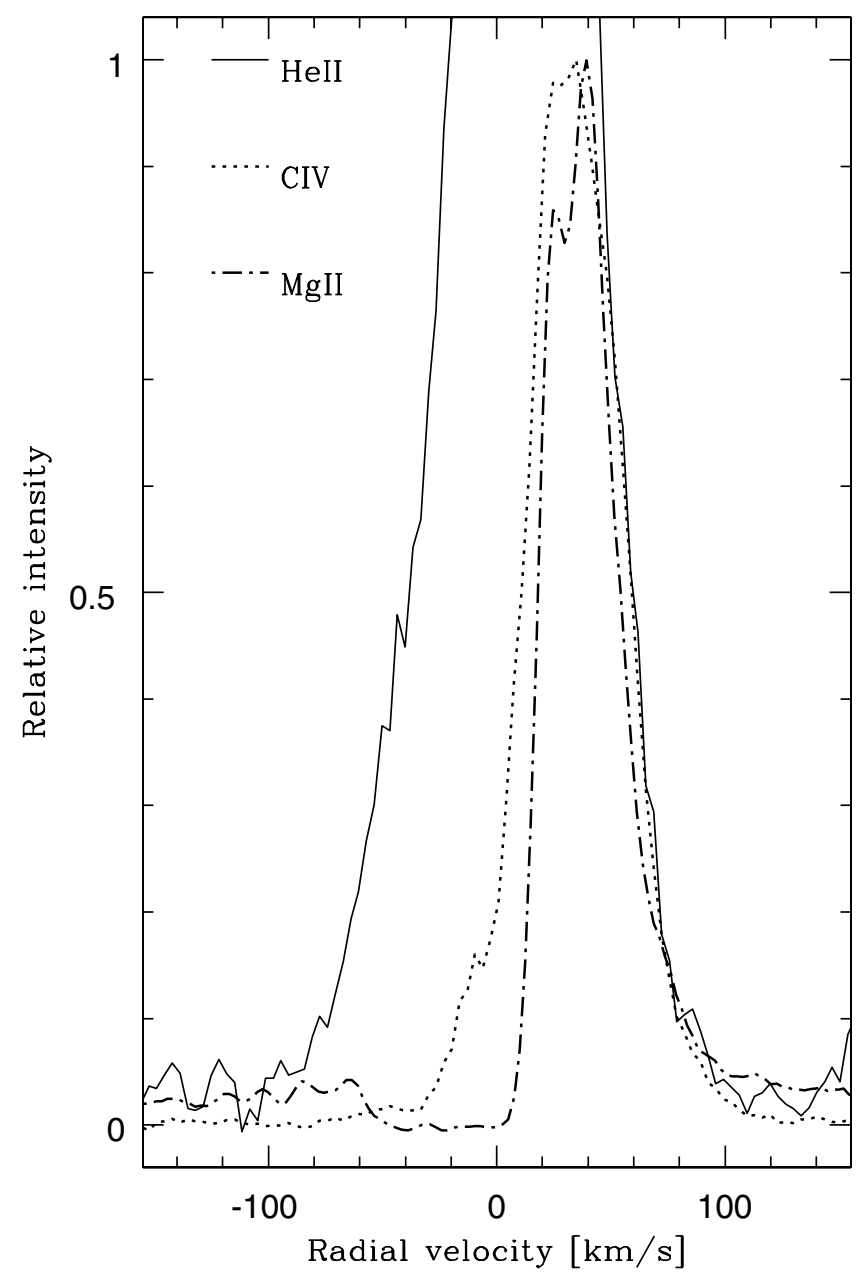

Fig. 3. The smoothened and normalized He II 1640 (solid line), average C IV profiles (dotted line) and Mg II (dashed line) profiles, observed with the HST/GHRS. The He II profile was increased by a factor of about 2, in order to fit the red wing to the C IV and Mg II profiles.

Keenan et al. 2002). Similarly, the S IV] 1416.9/1406.0 ratio indicates $n_{\mathrm{e}} \lesssim$ a few $\times 10^{9} \mathrm{~cm}^{-3}$ (Harper et al. 1999; Keenan et al. 2002). These electron densities are comparable to those derived for the He I, Balmer H I, and O III] emission region(s) by Kenyon et al. (1991). They may, however, not be representative of the resonance line emission region.

Let us examine the sizes of the regions of emission of the ultraviolet high ionization permitted zero volt lines of $\mathrm{C} \mathrm{IV}, \mathrm{N} \mathrm{V}$ and Si IV. These lines are expected to be excited by electron collisions. Assuming that the line emission is effectively optically thin, we can express the line luminosity as (e.g. Brown \& Jordan 1981; Mikołajewska et al. 1988):

$4 \pi \mathrm{d}^{2} F_{\lambda, \text { obs }}=8.6 \times 10^{-6} n_{\mathrm{e}} \frac{\Omega_{\mathrm{mn}}}{g_{\mathrm{m}}} \frac{N_{\mathrm{E}}}{N_{\mathrm{H}}} \frac{N_{\mathrm{H}}}{n_{\mathrm{e}}} \int_{\mathrm{V}} g(T) n_{\mathrm{e}}^{2} \mathrm{~d} V$

with

$g(T)=T_{\mathrm{e}}^{-1 / 2} \frac{N_{\text {ion }}}{N_{\mathrm{E}}} \exp \frac{-W_{\mathrm{mn}}}{k T_{\mathrm{e}}}$

Here $d$ is the distance, $F_{\lambda \text {,obs }}$ - the reddening-corrected observed line flux, $T_{\mathrm{e}}$ - the electron temperature, $N_{\mathrm{ion}}, N_{\mathrm{E}}, N_{\mathrm{H}}$ and $n_{\mathrm{e}}$ are the number densities of the ion in question, of an element $E$, of hydrogen, and electrons, respectively, $\Omega_{\mathrm{mn}}$ is the collision 


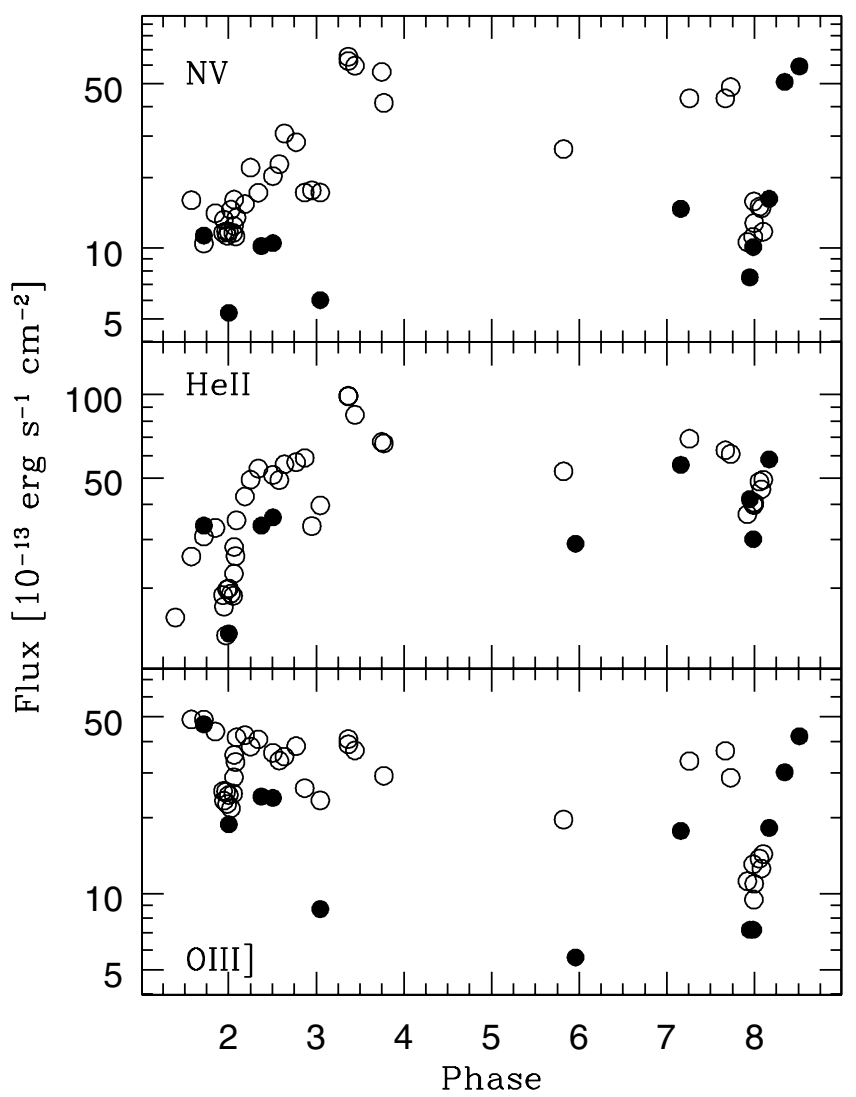

Fig. 4. Variation of line fluxes for N V 1240, He II 1640 and O III] 1664 emission lines. The open and filled circles correspond to the data from the high and low resolution IUE spectra, respectively.

Table 5. Emission measures, $E M$, in units of $10^{57} \mathrm{~cm}^{-3}$.

\begin{tabular}{cccccc}
\hline \hline MJD & Phase & N v & Si IV & C IV & Note \\
\hline 44831 & 0.506 & 140 & 31 & 22 & $(1)$ \\
& & 2.8 & 2.6 & 1.8 & $(2)$ \\
49271 & \multirow{2}{*}{0.696} & & 24 & 21 & $(1)$ \\
& & & 2.0 & 1.7 & $(2)$ \\
\hline
\end{tabular}

(1) $T_{\mathrm{e}}=15000 \mathrm{~K} ;(2) g(T)=0.7 g_{\max }(T)$.

strength of the transition from level $m$ to level $n, g_{\mathrm{m}}$ is the statistical weight of the lower level, and $W_{\mathrm{mn}}$ - the excitation energy.

The function $g(T)$ peaks strongly at a certain temperature, and it is often assumed $g(T)=0.7 g_{\max }(T)$ (Pottasch 1964). Such an assumption is valid for the line formation in a hot region, like the solar transition region, e.g. an accretion disk corona. Table 5 gives emission measures, $E M=\int_{V} n_{\mathrm{e}}^{2} \mathrm{~d} V$ calculated from the observed emission line fluxes, corrected for $E_{B-V}=0.4$ with the reddening curve of Seaton (1979), and adopting $d=2 \mathrm{kpc}$ (Mikołajewska \& Ivision 2001; Kenyon et al. 1991), for two values of $g(T): g(T)=0.7 g_{\max }(T)$ and $T_{\mathrm{e}}=15000 \mathrm{~K}$, representative of line formation in a hot shocked region and a photoionized region, respectively. We also assumed solar abundances (cf. Proga et al. 1996), and supposed that most of the atoms of the element in each line emission region were in the same state of ionization.

The present estimates take account of the fairly small contribution of the electrons due to ionization of helium, supposing it singly ionized in the $\mathrm{Si}^{+3}$ region and doubly ionized in the regions of $\mathrm{C}^{+3}$ and of $\mathrm{N}^{+4}$. The collision strengths used are those tabulated by Brown \& Jordan (1981), and Mikołajewska et al. (1988). In view of the presence of optical depth effects, we calculated the total rate of collisional excitation for each multiplet considered, assuming that photons were not lost by other sources of absorption.

It is noteworthy that if $T_{\mathrm{e}} \sim 15000 \mathrm{~K}$ the emission measure is $E M \sim$ a few $\times 10^{58} \mathrm{~cm}^{-3}$ for both the resonance lines and He II $1640 \AA$. For a density $n_{\mathrm{e}} \sim 10^{10} \mathrm{~cm}^{-3}$, typical for the central, eclipsed nebular region(s) in CI Cyg (e.g. Kenyon et al. 1991), emission measures of this order imply spherical radii lower than the binary separation, in agreement with some obscuration effects clearly visible in $\mathrm{N} \mathrm{V}$ lines.

We may note that such values of the emission measure are similar to the radio volume emission measure estimate of Mikołajewska \& Ivison (2001), but in view of the large length scale of the radio emission, of the order of $10^{15} \mathrm{~cm}$ (larger than the binary separation), this is presumably a coincidence. The radio emission region, however, can be the same as the resonance line absorbing region, if the optical thickness of the C IV lines is large.

\section{Discussion}

The fact the giant component of CI Cyg fills or nearly fills its tidal lobe implies that the usually adopted models for the symbiotic nebulae (with the main emission region in the spherically symmetric red giant wind ionised by the hot companion) cannot be a good match to the true conditions in the emission line region(s). The observed orbitally related changes of radial velocities and fluxes of most UV emission lines require the main formation region to be located inside the binary system. It is, however, in the partially ionised mass-loss stream and the environment of the accretion disk and the hot component rather than in a hemispherical shell just outside the red giant wind photosphere.

At the present stage, we cannot rule out completely radiative transfer as being responsible for the redshift of resonance lines with respect to the intercombination and He II lines. However a P Cygni profile explanation appears more likely, because of the lack of radial velocity changes of the resonance lines, suggesting that the lines are seriously affected by circumstellar absorption. He II $1640 \AA$ line is optically much thinner, as its lower level is at $41 \mathrm{eV}$ and is not affected. As far as the resonance line emission is concerned, our emission measures suggest line formation in regions smaller than the binary separation. The origin of the absorption component is however not obvious.

It cannot, in general, be due to interstellar line absorption (see discussion in Friedjung et al. 1983). However in the case of CI Cyg, two other objects in the same direction of the sky, show similar C IV (as well as Mg II) absorption to that suggested by the spectrum of CI Cyg. We see the absorption in high resolution IUE spectra of the X-ray binary Cygnus X-1 and the Wolf-Rayet star HD 190918, which are within $4^{\circ}$ from CI Cyg. This means that we cannot be certain to what extent the suggested CI Cyg absorption is really circumstellar. The absorption of HD 190918, spread out between very small negative radial velocities and about $-80 \mathrm{~km} \mathrm{~s}^{-1}$, has apparently no connection with the much more blueshifted absorption components, due to the wind of that star. However the spectra of the two other objects do not have $\mathrm{N} \mathrm{V}$ absorption. In fact the CI Cyg N v shift is at most only slightly less than the mean for the 6 high ionization lines $\left(2-7 \mathrm{~km} \mathrm{~s}^{-1}\right.$ on spectra where all 6 lines could be measured). This means that the redshift cannot be due to interstellar line absorption in the blue wings of the C IV doublet. 
The redshift cannot either be accounted by absorption by an iron curtain because the effect is present in all lines, including $\mathrm{N} V$ which should not be affected, whereas it is missing in the He II $1640 \AA$ line which should be affected by the $\mathrm{Fe}^{+}$-curtain (e.g. Shore \& Aufdenberg 1993).

Another possible effect is absorption of resonance line emission from excited Fe II levels, which results in pumping of highly excited levels of that ion and strong emission produced by cascades from these pumped levels. The emission of the C IV $1550 \AA$ and N V $1242 \AA$ lines is absorbed; if this absorption is optically thick, the profiles and relative fluxes of the lines in the same multiplet will be affected (Eriksson et al. 2003). However, according to Eriksson et al. (2006), no such pumping occurs for CI Cyg. The deviation of the C IV multiplet flux ratio from the optically thin value rather suggests self absorption. In any case the normalized profiles the C IV resonance multiplet lines are virtually identical, except for small noise.

The apparent absorption component should not occur in the same region as the emission. The absorbing region would need to be larger with a lower source function and would be presumably circumbinary. Such a region would need to be mainly in expansion, but some of the apparent absorption is to the red of the cool giant systemic velocity, as given by Kenyon et al. (1991). This means that parts of the region (inner parts) would need to be contracting towards the central binary. This might be just the gas pressure expansion velocity of a dense medium into a much lower density medium. Such a region might be due to the interaction between winds from the two binary components. Although Mikołajewska \& Ivison (2001) ruled out the interacting wind model (assuming spherically symmetric winds from both components) of Kenny (1995; see also Kenny \& Taylor 2005) as a possible mechanism for the extended radio emission from CI Cyg, we note that non spherically symmetric interacting winds are still possible. In particular, the mass loss from the giant is strongly concentrated in a stream which should result in the formation of a disk of material orbiting the companion. So, any wind from the companion should be bipolar rather than spherically symmetric. Moreover the disk itself can be also a source of a wind. The geometry of the mass flow(s) in CI Cyg thus differs significantly from the simple geometries assumed for the existing models. The resonance line absorption can be then located in the same region as the radio emission, for example, in a swept-up shell where a low-velocity material lost from the giant is overtaken by a higher velocity wind from the hot companion and/or accretion disk.

Let us note that short wavelength absorption is more clearly visible in ultraviolet spectra of other symbiotic binaries during outburst. A 1984 outburst spectrun of $\mathrm{Z}$ And shows two blueshifted components, one with a velocity of around $75-100 \mathrm{~km} \mathrm{~s}^{-1}$ and the other with an edge velocity of $230 \mathrm{~km} \mathrm{~s}^{-1}$ in C IV, while the lower velocity component is at least visible in $\mathrm{N}$ V. In particular, blueshifted absorption at $120 \mathrm{~km} \mathrm{~s}^{-1}$ in $\mathrm{N} \mathrm{V}$ and C IV was reported by Fernandez-Castro et al. (1995) for Z And at maximum. In addition blue shifted absorption has also been seen for $\mathrm{NV}$ when AG Dra was in outburst, the velocity being larger when the star was brighter (see Viotti et al. 1984).

The suggested ultraviolet P Cygni absorption is however not blueshifted enough to be produced by the wind of a compact object (lower main sequence star or more compact). Another possibility is that it is produced by the wind of an accretion disk around the compact component, which is slowly accelerated. Such a wind could have a much higher velocity than the observed radial velocity, because the disk would have a large inclination to the line of sight in this eclipsing system. Note that the blue shifted absorption components are probably not produced by a jet perpendicular to the orbital plane; any jet perpendicular to the orbital plane would be almost perpendicular to the line of sight and not absorbing.

The He II line blueshift is explainable if the emission is formed in an expanding medium above the central regions of an optically thick accretion disk The similarity of the intercombination line orbit to that of the optical Fe II orbit might be understandable if the Fe II lines were optically thin and formed near the intercombination line region. Kenyon et al (1991) explained both the apparent eccentricity of the orbit and the phase displacement of the spectroscopic conjunction by the formation of Fe II near the inner Lagrangian point in the gas stream and/or bright spot where the matter stream impacts the outer accretion disk. The small difference in radial velocity amplitudes and phase shifts between the intercombination and Fe II lines can in principle be explained by some stratification effects in the stream. The change between decline and quiescence might be related to a change in the properties of the disk.

\section{Conclusions}

Archival ultraviolet spectra taken by IUE and at higher resolution at one epoch by the GHRS/HST are studied by us. We examined radial velocities and line fluxes as well as higher resolution HST line profiles, taken at one epoch. The line fluxes were used to determine electron densities and emission measures; line formation in regions rather smaller than the binary separation being indicated.

The relative systemic velocity shifts of the He II and intercombination lines may be explicable by expansion, possibly in a medium above an optically thick accretion disk, with stratification. In this framework changes in disk properties might be responsible for changes in the intercombination line radial velocity between decline and quiescence.

A systematic redshift between the optically thick resonance lines on the one hand and the optically thin intercombination lines and the usually not optically very thick He II $1640 \AA$ line on the other hand, like that of Friedjung et al. (1983), was confirmed by us. Two possible explanations still exist. We favour that involving a non-classical P Cygni profile due to large circumbinary absorbing region, which is mainly expanding, but of which parts (perhaps inner parts) are contracting towards the binary. This region is most probably an asymmetric wind interaction shell or a wind from the accretion disk. Such a region could also produce the observed radio emission. The other explanation of the redshift, as due to radiative transfer effects, cannot yet however be completely eliminated. More theoretical work on the production of such a line shift is still required.

Acknowledgements. This research has been partly supported by KBN grants 5P03D 019 20, and 1P03D 017 27, and by the European Associated Laboratory "Astrophysics Poland-France". It also made use of the NASA Astrophysics Data System and SIMBAD database.

\section{References}

Belczyński, K., Mikołajewska, J., Munari, U., Ivison, R. J., \& Friedjung, M. F. 2000, A\&AS, 146, 407

Brown, A., \& Jordan, C. 1981, MNRAS, 196, 757

Eriksson, M., Johansson, S., \& Wahlgren, G. M. 2003, A\&A, 422, 987

Eriksson, M., Johanssen, S., \& Wahlgren, G. M. 2006, A\&A, 451, 157

Fernandez-Castro, T., Gonzalez-Riestra, R., Cassatella, A., Taylor, A. R., \& Seaquist, E. R. 1995, ApJ, 442, 366

Friedjung, M., Stencel, R. E., \& Viotti, R. 1983, A\&A, 126, 407 
Harper, G. M., Jordan, C., Judge, P. G., et al. 1999, MNRAS, 303, L41

Kastner, S. O., Bhatia, A. K., \& Feibelman, W. A. 1989, MNRAS, 237, 487 Keenan, F. P., Ahmed, S., Brage, T. et al. 2002, MNRAS, 337, 901

Kenny, H. T. 1995, Ph.D. Thesis, Univ. Calgary

Kenny, H. T., \& Taylor, A. R. 2005, ApJ, 619, 527

Kenyon, S. J., Oliversen, N. A., Mikołajewska, J., et al. 1991, AJ, 101, 637

Mikołajewska, J. 2001, in Small Telescope Astronomy on Global Scales, ed. B. Paczynski, W. P. Chen, C. Lemme, ASP Conf. Ser., 246, 167

Mikołajewska, J. 2003, in Symbiotic Stars Probing Stellar Evolution, ed. R. L. M. Corradi, J. Mikolajewska, T. J. Mahoney, ASP Conf. Ser. 303, 9

Mikołajewska, J., \& Ivison, R. J. 2001, MNRAS, 324, 1023

Mikołajewska, J., \& Friedjung, M. 2005, Ap\&SS, 296, 441
Mikołajewska, J., Selvelli, P. L. \& Hack, M. 1988, A\&A, 198, 150

Mürset, U., \& Schmid, H. M. 1999, A\&AS, 137, 473

Nussbaumer, H., \& Storey, P. J. 1979, A\&A, 71, L5

Nussbaumer, H., \& Storey, P. J. 1982, A\&A, 115, 205

Nussbaumer, H., \& Schmutz, H. 1983, A\&A, 126, 59

Pottasch, S. R. 1964, Space Sci. Rev., 3, 816

Proga, D., Kenyon, S. J., Raymond, J. C., \& Mikołajewska, J. 1996, ApJ, 471, 930

Seaton, M. J. 1979, MNRAS, 187, 73p

Shore, S., \& Aufdenberg, J. 1993, ApJ, 416, 355

Viotti, R., Altamore, A., Baratta, G. B., Cassatella, A., \& Friedjung, M. 1984, ApJ, 283, 226 